\title{
Vancomicina en infusión continua en pacientes pediátricos críticos
}

\author{
Continuous infusion of vancomycin in pediatric critical care
}

\author{
Dra. Brenda Lara Zylbersztajn ${ }^{a}$,Dra. Pamela Chicco ${ }^{a}$ Dra. Laura Vega ${ }^{a}$, Dra. Mónica Centeno ${ }^{a}$ \\ Dra. Silvia Filippini ${ }^{a}$ y Dra. Silvina Ruvinsky ${ }^{a}$
}

\begin{abstract}
RESUMEN
La administración de vancomicina en infusión continua es una estrategia de tratamiento posible en pacientes críticos que no alcancen niveles plasmáticos adecuados. Existe escasa bibliografía acerca de este tipo de administración.

Se presentan 6 niños ( 2 meses a 7 años; 4 varones y 2 mujeres) que ingresaron en la unidad de cuidados intensivos del Hospital de Pediatría Garrahan con un cuadro clínico de sepsis por Staphylococcus aureus resistente a la meticilina, tratados con vancomicina, en dosis de entre 40 y $60 \mathrm{mg} / \mathrm{kg} /$ día cada 8-6 horas. Debido a la evolución clínica no favorable, la persistencia de la fiebre, los cultivos positivos y los niveles plasmáticos del antibiótico insuficientes, se implementó la infusión continua a 50 $\mathrm{mg} / \mathrm{kg} /$ día. Todos los pacientes alcanzaron niveles entre 10 y $25 \mu \mathrm{g} / \mathrm{ml}$, evolucionaron favorablemente y negativizaron los cultivos, sin signos de nefrotoxicidad. El tiempo de tratamiento en infusión continua fue entre 9 y 18 días.

La infusión continua de vancomicina fue eficaz en estos pacientes, sin evidencias de nefrotoxicidad asociada.

Palabras clave: vancomicina, pediatría, cuidados críticos, infusión continua.
\end{abstract}

\section{SUMMARY}

Continuous infusion of vancomycin may be a strategy for critically ill patients who do not achieve adequate plasma levels. There is few literature on this dosage regimen.

We present six children ( 2 months to 7 years, 4 male and $2 \mathrm{fe}-$ male), admitted to the Intensive Care Unit of the "Prof. Dr. Garrahan Children Hospital", with methicillin-resistant Staphylococcus aureus sepsis, treated with vancomycin 40 and $60 \mathrm{mg} /$ $\mathrm{kg} /$ day every 8-6 hrs. Continuous infusion at $50 \mathrm{mg} / \mathrm{kg} /$ day was implemented due to poor outcome, persistent fever, positive cultures and inadequate vancomycin plasma levels. All patients achieved levels between 10 and $25 \mathrm{ug} / \mathrm{ml}$, their outcome was favorable and cultures became negative, with no signs of nephrotoxicity. Treatment duration of the continuous infusion was 9 to 18 days.

Continuous infusion of vancomycin was effective in these patients without evidence of associated nephrotoxicity.

Key words:vancomycin, pediatric, critical care, continuous infusion.

http:/ /dx.doi.org/10.5546/aap.2012.e31

a. Hospital de Pediatría "Prof. Dr. Juan P. Garrahan".

\section{Correspondencia:}

Dra. Brenda Lara Zylbersztajn:

brenda.zylbersztajn@gmail.com

Conflicto de intereses: Ninguno que declarar.

Recibido: 7-8-2012.

Aceptado: 25-9-2012.

\section{INTRODUCCIÓN}

La vancomicina es un antibiótico glucopéptido, cuyo uso se ha incrementado debido al aumento de la prevalencia de microorganismos grampositivos resistentes, incluidas cepas de Staphylococcus aureus resistentes a la meticilina (SARM) y enterococos resistentes a la penicilina.

Su actividad máxima se obtiene al mantener una relación área bajo la curva/concentración inhibitoria mínima del patógeno mayor de $400 .{ }^{1}$ En los pacientes críticos, la farmacocinética del principio activo puede alterarse, impidiendo alcanzar las concentraciones recomendadas, por lo que una estrategia de tratamiento sería la administración en infusión continua. Esta modalidad permite mantener niveles constantes del antibiótico en el plasma y favorece una mayor penetración en los tejidos. ${ }^{2-4}$

Es escasa la información acerca de la eficacia y la toxicidad de este tipo de administración..$^{5-8}$

Se presentan 6 pacientes ingresados en la unidad de cuidados intensivos (UCI) del Hospital de Pediatría "Prof. Dr. J. P. Garrahan" que recibieron infusión continua de vancomicina.

En todos los casos la determinación de vancomicina en el plasma se realizó por inmunofluorescencia polarizada (TDx Abbott), límite de detección de $2 \mu \mathrm{g} / \mathrm{ml}$ y se evaluó la función renal por el valor de creatinina sérica.

\section{CASOS CLÍNICOS}

1. Niño de 2 meses, con diagnóstico de linfangioma cérvico-torácico, que ingresó en la UCI tras requerir una traqueostomía de urgencia. Días después, presentó un cuadro de sepsis, confirmándose tromboflebitis supurada con aislamiento en hemocultivos de SARM. Se inició tratamiento con vancomicina ( $40 \mathrm{mg} / \mathrm{kg} /$ día cada 8 horas).

Se realizaron determinaciones de concentración plasmática en el valle de vancomicina, con ajustes posteriores de la dosis hasta $80 \mathrm{mg} / \mathrm{kg}$ / día cada 6 horas, sin evidencia de mejoría clínica ni obtención de niveles plasmáticos adecuados (5,05 y $9,95 \mu \mathrm{g} / \mathrm{ml})$. 
Debido a la gravedad del cuadro y a la sepsis con bacteriemia persistente por SARM, después de 12 días de tratamiento se instauró la infusión continua de vancomicina a $50 \mathrm{mg} / \mathrm{kg} /$ día, obteniéndose niveles séricos entre 9,89 y 13,77 ug/ml. Al quinto día de iniciada esta, se logró la negativización de los hemocultivos y la mejoría clínica. El esquema se mantuvo durante 16 días y continuó con la administración intermitente a $60 \mathrm{mg} / \mathrm{kg} /$ día cada 6 horas. No se observó deterioro de la función renal. Continuó con los controles ambulatorios y evolución clínica favorable.

2. Niño de 7 años que ingresó en la UCI por sepsis con foco osteoarticular en el miembro inferior izquierdo y neumonía necrosante con supuración pleuropulmonar por SARM que requirió asistencia ventilatoria mecánica (AVM) e inotrópicos. Se inició tratamiento con vancomicina ( $40 \mathrm{mg}$ / $\mathrm{kg} /$ día cada 8 horas), rifampicina y trimetoprimasulfametoxazol, y se realizó el drenaje quirúrgico de las colecciones osteoarticulares y pleurales. Se implementaron sucesivos ajustes en la dosis de vancomicina, alcanzando niveles de $9,18 \mu \mathrm{g} / \mathrm{ml}$ con $80 \mathrm{mg} / \mathrm{kg} /$ día cada 6 horas. Debido a la persistencia de los cultivos positivos, la inestabilidad clínica y los niveles subterapéuticos de vancomicina, el día 9 de tratamiento se inició la infusión continua del antibiótico a $50 \mathrm{mg} / \mathrm{kg} /$ día, obteniendo niveles entre 13,73 y 22,52 $\mathrm{gg} / \mathrm{ml}$. En los días posteriores, el niño presentó una franca mejoría, se negativizaron los cultivos y después de 14 días de administración continua se cambió al régimen intermitente. Con una evolución clínica favorable, posteriormente se le otorgó el alta y continuó con los controles ambulatorios.

3. Niño de 11 meses que ingresó en la UCI con un cuadro de shock séptico y dificultad respiratoria que requirió AVM e inotrópicos. Con diagnóstico de neumonía necrosante con supuración pleuropulmonar por SARM, se inició tratamiento con vancomicina $15 \mathrm{mg} / \mathrm{kg}$ cada 8 horas. Al no observar respuesta al tratamiento y por la progresión del cuadro clínico se agregó trimetoprimasulfametoxazol y rifampicina.

Debido a la persistencia de niveles inadecuados de vancomicina $(7,9-8,38 \mu \mathrm{g} / \mathrm{ml})$, al décimo día de tratamiento se inició la infusión continua de vancomicina a $50 \mathrm{mg} / \mathrm{kg} /$ día alcanzando niveles adecuados $(17,46-25,45 \mu \mathrm{g} / \mathrm{ml})$. Progresivamente, el niño comenzó a mejorar y el tratamiento en infusión continua se mantuvo por 17 días. Completó el tratamiento con trimetoprima-sulfa- metoxazol y rifampicina por vía oral, con evolución clínica favorable y posterior alta hospitalaria.

4. Niña de 7 años que ingresó en la UCI por un traumatismo, con un cuadro de shock séptico asociado a SARM, requerimiento de inotrópicos y AVM. Se inició tratamiento con vancomicina $60 \mathrm{mg} / \mathrm{kg} /$ día cada 6 horas y rifampicina. Conjuntamente presentó derrame pericárdico y pleural que requirió el drenaje de las colecciones. Por los hemocultivos positivos persistentes y los niveles subterapéuticos de vancomicina, al día 5 de tratamiento se aumentó la dosis a $80 \mathrm{mg} / \mathrm{kg} /$ día cada 6 horas.

Al día 8 de tratamiento, debido a la evolución desfavorable y la persistencia de concentraciones plasmáticas bajas del antibiótico, se inició la infusión continua de vancomicina a $50 \mathrm{mg} / \mathrm{kg} /$ día. Los valores séricos de vancomicina fueron cercanos a $14 \mu \mathrm{g} / \mathrm{ml}$, por cual se decidió aumentar la dosis de la infusión continua a $60 \mathrm{mg} / \mathrm{kg} /$ día. Posteriormente la paciente presentó buena evolución clínica y se lograron los niveles adecuados del fármaco.

Después de 18 días de infusión continua, completó el tratamiento por vía oral con rifampicina y clindamicina, con el egreso hospitalario posterior.

5. Niño de 6 años, que ingresó en la UCI con shock séptico y púrpura fulminante por SARM, requiriendo $\mathrm{AVM}$ e inotrópicos. Se inició tratamiento con vancomicina $(15 \mathrm{mg} / \mathrm{kg}$ cada 6 horas) y clindamicina, agregándose posteriormente rifampicina. Por la persistencia de niveles bajos $(2-6,03 \mu \mathrm{g} / \mathrm{ml})$ en el valle de vancomicina y la persistencia de la fiebre, al sexto día de tratamiento se colocó vancomicina en infusión continua a $50 \mathrm{mg} / \mathrm{kg}$ / día y se logró alcanzar niveles adecuados en los controles posteriores $(20,88 \mu \mathrm{g} / \mathrm{ml})$. Presentó una notoria mejoría clínica y al noveno día de tratamiento con cultivos negativos pasó a la sala general, completando 14 días de infusión continua y egreso hospitalario posterior.

6. Niña de 22 meses, que ingresó en la UCI con un cuadro de shock séptico secundario a fascitis necrosante por SARM en el miembro inferior izquierdo. Comenzó tratamiento con vancomicina (40 mg/kg/día cada 8 horas) ceftriaxona y clindamicina, y se realizó desbridamiento quirúrgico. La niña requirió AVM e inotrópicos. Por la gravedad del cuadro y los niveles insuficientes de vancomicina se incrementó la dosis en forma escalonada hasta alcanzar $80 \mathrm{mg} / \mathrm{kg} /$ día. Durante la admi- 
nistración intermitente, las concentraciones plasmáticas del antibiótico fueron 4,97 y 6,89 $\mu \mathrm{g} / \mathrm{ml}$. El día 9 de tratamiento, por la mala evolución y la persistencia de la fiebre, se cambió a infusión continua a $50 \mathrm{mg} / \mathrm{kg} /$ día. Al cuarto día de iniciada esta modalidad de tratamiento la paciente tuvo una franca mejoría y negativización de los cultivos. Los niveles alcanzados fueron entre 16,03 y $21,31 \mu \mathrm{g} / \mathrm{ml}$. La infusión continua se mantuvo durante 8 días y continuó el tratamiento con trimetoprima-sulfametoxazol y rifampicina por vía oral.

Las concentraciones plasmáticas de los seis pacientes y los respectivos períodos de infusión continua se muestran en la Figura 1.

\section{COMENTARIOS}

En la población adulta se observó una eficacia clínica similar al comparar la administración de vancomicina intermitente y continua. $5,6,9,10$

Con la emergencia de las infecciones graves por SARM adquiridas en la comunidad, se incorporó la recomendación de alcanzar niveles séricos de vancomicina en el valle de $15-20 \mu \mathrm{g} / \mathrm{ml}^{1}$ Cabe aclarar que el primer paciente fue tratado antes de las nuevas recomendaciones, por lo que las concentraciones buscadas fueron menores que en el resto.

Las dosis iniciales empleadas en el régimen de infusión continua fueron superiores a las mencionadas en otros estudios provenientes, en su mayoría, de una población adulta. Se debe considerar que la población blanco de nuestro estudio fueron niños, los cuales presentan características farma- cocinéticas diferentes y requieren, en ocasiones, esquemas terapéuticos diferentes. ${ }^{11}$

Mediante la infusión continua se logró alcanzar niveles adecuados de vancomicina y controlar la infección de los pacientes presentados sin evidencias de lesión renal. La infusión continua de vancomicina sería una estrategia terapéutica para considerar en los pacientes críticos con infección grave por SARM que no logran alcanzar niveles adecuados del fármaco en el plasma.

Se necesitan estudios pediátricos seleccionados al azar y controlados que evalúen la eficacia y seguridad de esta forma de administración.

\section{BIBLIOGRAFÍA}

1. Rybak MJ, Lomaestro BM, Rotschafer JC, Moellering RC, et al. Vancomycin therapeutic guidelines: a summary of consensus recommendations from the Infectious Diseases Society of America, the American Society of Health-System Pharmacists, and the Society of Infectious Diseases Pharmacists. Clin Infect Dis 2009;49(3):325-7.

2. Alonso-Fernández MA, Estébanez-Montiel MB, Rico-Cepeda MP, Catalan-González M, et a. Vancomicina en perfusión continua, una nueva pauta posológica en la Unidad de Cuidados Intensivos. Med Intensiva 2005;29(2):83-7.

3. Albanese J, Léone M, Bruguerolde B, Ayem ML, et al. Cerebrospinal fluid penetration and pharmacokinetics of vancomycin administered by continuous infusion to mechanically ventilated patients in an Intensive Care Unit. Antimicrob Agents Chemother 2000;44(5):1356-8.

4. Byl B, Jacobs F, Wallemacq P, Rossi C, et al. Vancomycin penetration of uninfected pleural fluid exudate after continuous or intermittent infusion. Antimicrob Agents Chemother 2003;47(6):2015-7.

5. Plan O, Cambonie G, Barbotte E, Meyer P, et al. Continuous-infusion vancomycin therapy for preterm neonates with suspected or documented Gram-positive infections:

FIGURA 1. Concentraciones plasmáticas de vancomicina en función del tiempo de tratamiento

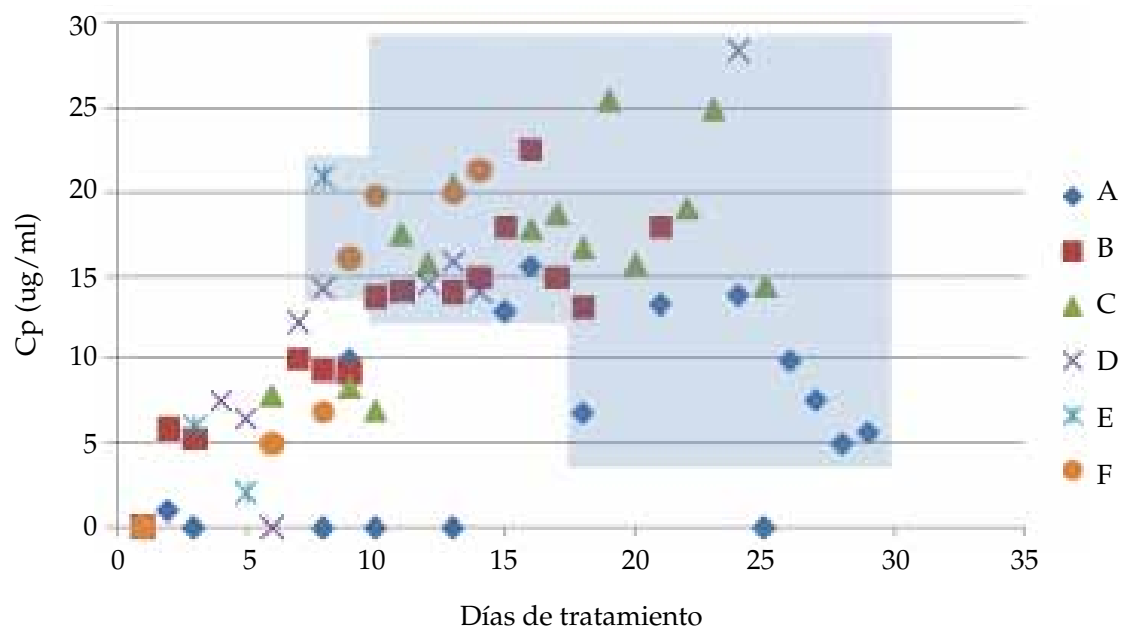

El área sombreada corresponde al período de infusión continua de los pacientes. 
a new dosage schedule. Arch Dis Child Fetal Neonatal 2008;93(6):F418-21.

6. EmbletonN, BerringtonJ.Giving vancomycin as a continuous infusion. Arch Dis Child Fetal Neonatal 2009;94(3):F233-4.

7. Ingram P, Lye D, Fisher D, Goh W, Tam V. Nephrotoxicity of continuous versus intermittent infusion of vancomycin in outpatient parenteral antimicrobial therapy. Int J Antimicrob Agents 2009;34(6):570-4.

8. Saugel B, Nowack MC, Hapfelmeier A, Umgelter A, et al. Continuous intravenous administration of vancomycin in medical intensive care unit patients. J Crit Care 2013; 28(1):9-13.
9. Lacy M, Tessier P, Nicolau D, Nightingale C, Quintiliani R. Comparison of vancomycin pharmacodynamics (1 g every 12 or $24 \mathrm{hr}$ ) against methicillin-resistant staphilococci. Int J Antimicrob Agents 2000;15(1):25-30.

10. Cataldo MA, Tacconelli E, Grilli E, Pea F, Petrosillo N. Continuous versus intermittent infusion of vancomycin for the treatment of Gram-positive infections: systematic review and meta-analysis. J Antimicrob Chemother 2012,67(1):17-24.

11. Dehority W. Use of vancomycin in pediatrics. Pediatr Infect Dis J 2010;29(5):462-4. 